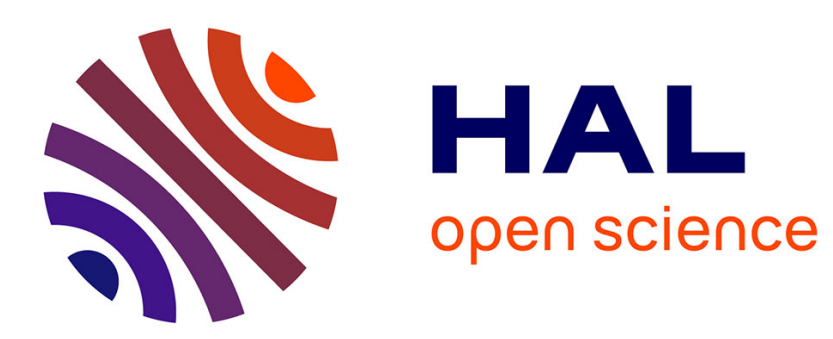

\title{
Discrete event system identification with the aim of fault detection
}

\author{
Marcos V Moreira, Jean-Jacques Lesage
}

\section{To cite this version:}

Marcos V Moreira, Jean-Jacques Lesage. Discrete event system identification with the aim of fault detection. Discrete Event Dynamic Systems: Theory and Applications, 2019, 10.1007/s10626-01900283-z . hal-02171280

\section{HAL Id: hal-02171280 \\ https://hal.science/hal-02171280}

Submitted on 2 Jul 2019

HAL is a multi-disciplinary open access archive for the deposit and dissemination of scientific research documents, whether they are published or not. The documents may come from teaching and research institutions in France or abroad, or from public or private research centers.
L'archive ouverte pluridisciplinaire HAL, est destinée au dépôt et à la diffusion de documents scientifiques de niveau recherche, publiés ou non, émanant des établissements d'enseignement et de recherche français ou étrangers, des laboratoires publics ou privés. 


\title{
Discrete event system identification with the aim of fault detection
}

\author{
Marcos V. Moreira · Jean-Jacques Lesage
}

the date of receipt and acceptance should be inserted later

\begin{abstract}
In this paper, we present a method for discrete event system identification with the aim of fault detection. The method is based on a new model called Deterministic Automaton with Outputs and Conditional Transitions (DAOCT), which is computed from observed fault-free paths, and represents the fault-free system behavior. In practice, a trade-off between size and accuracy of the identified automaton has to be found. In order to obtain compact models, loops are introduced in the model, which implies that sequences that are not observed can be generated by the model leading to an exceeding language. This exceeding language is associated with possible non-detectable faults, and must be reduced in order to use the model for fault detection. We show, in this paper, that the exceeding language generated by the DAOCT is smaller than the exceeding language generated by another model proposed in the literature, reducing, therefore, the number of possible non-detectable faults. We also show that if the identified DAOCT does not have cyclic paths, then the exceeding language is empty, and the model represents all and only all observed fault-free sequences generated by the system. In order to illustrate the results of the paper, a physical system is simulated by using a 3D simulation software controlled by a Programmable Logic Controller (PLC). The main idea is to use a virtual digital system to simulate the fault-free behavior of a physical system, captured by the sequences of input and output signals of the PLC, and then use the method proposed in the paper to obtain the DAOCT model of the plant.
\end{abstract}

\section{Introduction}

Fault detection and isolation has received considerable attention from the scientific community over the last years. In Sampath et al. (1995), a discrete-event approach for fault diagnosis is introduced, and since then, several works have been proposed for fault detection and isolation, and also for the verification of diagnosability of the system, i.e., the capability of identifying the occurrence of a fault event within a bounded number of occurrences of events (Debouk et al., 2000; Qiu and Kumar, 2006; Moreira et al., 2011; Carvalho et al.,

Marcos V. Moreira, E-mail: moreira.mv@poli.ufrj.br Department of Electrical Engineering, Universidade Federal do Rio de Janeiro, 21949-900, Rio de Janeiro, R.J, Brazil.

Jean-Jacques Lesage, E-mail: jean-jacques.lesage@ens-paris-saclay.fr

LURPA, ENS Cachan, Univ. Paris-Sud, Université Paris-Saclay, 94235 Cachan, France. 
2012, 2013; Zaytoon and Lafortune, 2013; Cabral et al., 2015b,a; Cabral and Moreira, 2017; Santoro et al., 2017). In all these works, it is assumed that the complete system behavior is known, i.e., the system behavior before and after the occurrence of fault events.

Although methods for fault detection based on the complete system behavior can be successfully applied to small systems, they are difficult to be implemented on large and complex systems for the following reasons: $(i)$ in general, large automated systems are composed of several components, whose models and interactions between these models, are difficult or even impossible to be obtained; (ii) the modeling process requires engineers that know the complete plant behavior, and are also familiar with discrete-event modeling techniques; (iii) the post-fault behavior of the system is difficult to be predicted due its size and complexity; and (iv) only faults that have been predicted can be detected by the diagnoser computed considering the complete behavior of the system.

In order to overcome the problem of modeling DES, several works in the literature propose identification methods based on automata or Petri nets for different purposes (Medhi et al., 2006; Cabasino et al., 2007; Lefebvre and Delherm, 2007; Dotoli et al., 2008; EstradaVargas et al., 2010; Dotoli et al., 2011; Estrada-Vargas et al., 2014a,b; Cabasino et al., 2013; Estrada-Vargas et al., 2015). The majority of these works address the problem of identifying Petri net models that are not suitable for fault diagnosis. A method for the identification of a Petri net model suitable for fault diagnosis is proposed in Cabasino et al. (2014). In Cabasino et al. (2014), the faulty behavior of the system is identified based on the observation of the events generated by the system, and it is assumed that the fault-free model is known. Thus, the method proposed in Cabasino et al. (2014) does not address the problem of obtaining large and complex fault-free models of DES. In Dotoli et al. (2008), a Petri net model for a DES is obtained assuming that all events are observable. Although in Dotoli et al. (2008) it is not necessary to consider that all fault-free model is known, it is assumed that some state components are known, and an upper bound on the cardinality of the place set is given. The existence of unobservable events is considered in Dotoli et al. (2011) for system identification using Petri nets. In Dotoli et al. (2011), it is considered that the faulty behavior of the system is unknown, and the problem of identifying the unobservable behavior of a DES is addressed. In order to do so, the fault-free system structure and dynamics are assumed to be known.

Fault detection techniques based on an identified fault-free model of the system have been recently proposed (Klein et al., 2005; Roth et al., 2009, 2011). Differently from Dotoli et al. (2011), in these works the fault-free system structure and dynamics are not known, and the two main ideas are: (i) to automate the process of obtaining the fault-free model of the system by using identification; and (ii) when a fault has been detected through a discrepancy between the system behavior and the model, to use a technique based on residuals for fault isolation. This strategy is depicted in Figure 1. In Klein et al. (2005), a monolithic model for fault detection, that is capable of representing the behavior of a closed-loop system, is proposed. This model is non-deterministic with state outputs, and has been called NonDeterministic Autonomous Automaton with Output (NDAAO). The NDAAO is obtained from observed sequences of binary signals exchanged between the plant and the controller (sensor signals emitted by the plant and actuator commands generated by the controller), as shown in Figure 2. In Klein et al. (2005), it is shown that the identified NDAAO generates all observed sequences of signals used in the identification process. Furthermore, a tradeoff between size and accuracy of the identified model can be found thanks to an adequate adjustment of the parametric algorithm used for identification. Indeed, for reducing the size of the model, equivalent states are merged, what introduces loops in the NDAAO, generating sequences that have not been observed. This exceeding language can increase the number 


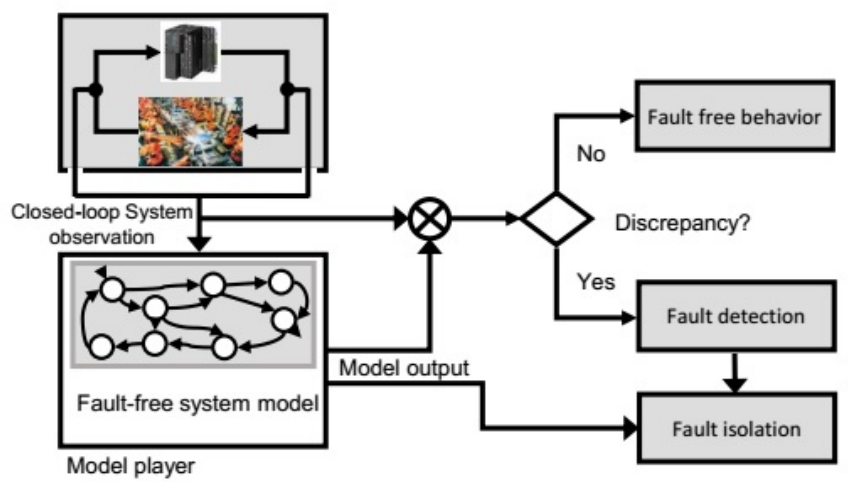

Fig. 1: Model-based diagnosis based on a fault-free model.

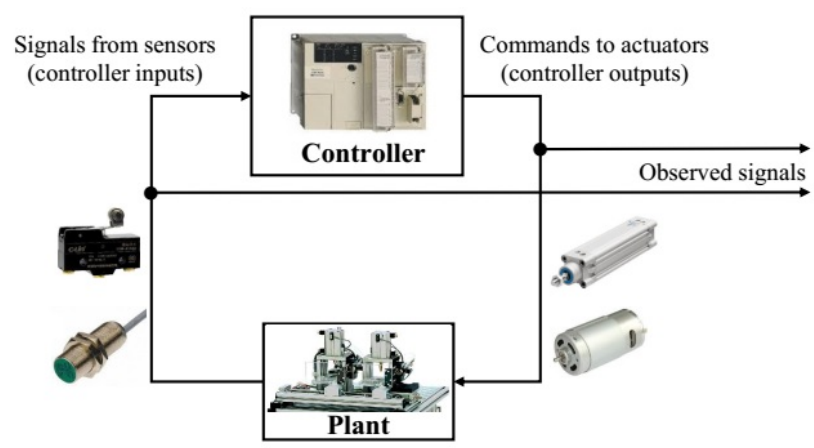

Fig. 2: Closed-loop discrete-event system.

of non-detectable faults of the system, and may prevent the fault detection scheme to be implemented. In order to deal with this trade-off, in Klein et al. (2005), a free parameter $k$, that is used to compute the NDAAO, is introduced, and it is shown that the NDAAO is $k+1$-complete in the sense of Moor et al. (1998), i.e., a sequence of signals of length smaller than or equal to $k+1$ belongs to the identified NDAAO if, and only if, it is observed in the system.

In Roth et al. (2009) and Roth et al. (2011), the fault detection strategy proposed in Klein et al. (2005) is extended to systems with a high degree of concurrency. As in Klein et al. (2005), the NDAAO is used, and the same trade-off between model size and accuracy is observed in these works.

In this paper, we present a new model for discrete-event system identification that is more efficient for fault detection than the method proposed in Klein et al. (2005) and Roth et al. (2009, 2011), called Deterministic Automaton with Outputs and Conditional Transitions (DAOCT). In order to do so, the same strategy for fault detection, depicted in Figure 1, and the same assumptions presented in Klein et al. (2005) are considered in this work. The 
exceeding language generated by the DAOCT is reduced in comparison with the exceeding language generated by the NDAAO, due to a path estimation function that is added to the model, reducing the number of non-detectable faults. We also show that if the identified DAOCT does not have cyclic paths, then there is no exceeding language. As in Klein et al. (2005), we assume that the binary input and output signals of the controller are measured, generating the observed fault-free paths of the system. Using this information, the DAOCT is computed. The DAOCT also satisfies the property of $k+1$-completeness, if sequences of observed signals are considered, or, equivalently, $k$-completeness if sequences of events are considered, i.e., a sequence of events of length $k$ belongs to the identified model if, and only if, it belongs to an observed path of the system.

This paper is an extended version of the work presented in Moreira and Lesage (2018), where we have introduced the DAOCT model for DES identification. In this paper, differently from Moreira and Lesage (2018), we illustrate the results by using a physical system simulated in a 3D simulation software controlled by a Programmable Logic Controller (PLC). The main idea is to use the digital twin of a physical system to simulate its fault-free behavior, captured by the sequences of input and output signals of the PLC, and then use the method proposed in the paper to obtain the DAOCT model of the plant. The use of digital twins of physical plants is growing in importance for analysis and design of complex systems such as mechatronic systems (Boschert and Rosen, 2016; Grieves and Vickers, 2017), manufacturing systems (Schleicha et al., 2017; Uhlemann et al., 2017), and airspace vehicles (Glaessgen and Stargel, 2012). In this paper, we use a digital plant for identification of a DES with the aim of fault detection.

This paper is organized as follows. In Section 2, we present some preliminary concepts and the basic ideas of fault detection based on the fault-free behavior of the system. In Section 3, we formulate the problem of system identification with the aim of fault detection, and in Section 4, we introduce the DAOCT model for system identification. In Section 5, we present the digital plant used to illustrate the results of the paper. Finally, in Section 6, the conclusions are drawn.

\section{Preliminaries}

\subsection{Notation and Definitions}

Let $G=\left(X, \Sigma, f, x_{0}, X_{m}\right)$ denote a deterministic automaton (Cassandras and Lafortune, 2008), where $X$ is the set of states, $\Sigma$ is the finite set of events, $f: X \times \Sigma^{\star} \rightarrow X$ is the transition function, where $\Sigma^{\star}$ is the Kleene-closure of $\Sigma, x_{0}$ is the initial state of the system, and $X_{m}$ is the set of marked states.

The language generated by $G$ is defined as $L(G)=\left\{s \in \Sigma^{\star}: f\left(x_{0}, s\right) !\right\}$, where ! denotes is defined. The prefix-closure of a language $L$ is defined as $\bar{L}=\left\{s \in \Sigma^{\star}:\left(\exists t \in \Sigma^{\star}\right)(s t \in L)\right\}$. Notice that the language generated by $G$ is prefix-closed by definition.

The function of feasible events $\Gamma: X \rightarrow 2^{\Sigma}$, is defined as $\Gamma(x)=\{\sigma \in \Sigma: f(x, \sigma) !\}$.

The set of all subsequences of a sequence $s \in \Sigma^{\star}$ is defined as $\operatorname{Sub}(s)=\left\{w \in \Sigma^{\star}\right.$ : $\left.\left(\exists t, w, v \in \Sigma^{\star}\right)(s=t w v)\right\}$.

A path $p$ of an automaton $G$ is a sequence of states and events that can be executed by the system, i.e., a path $p=\left(x_{1}, \sigma_{1}, x_{2}, \sigma_{2}, \ldots, \sigma_{l-1}, x_{l}\right)$ is feasible in $G$ if, and only if, $x_{i} \in X$, for $i=1,2, \ldots, l, \sigma_{i} \in \Sigma$, for $i=1,2, \ldots, l-1$, and $f\left(x_{i}, \sigma_{i}\right)=x_{i+1}, i=1, \ldots, l-1$. The length of a path is defined as the number of vertices in the path, and is denoted here as $\|p\|$. Thus, $\|p\|=l$. A path is said to be cyclic if $x_{l}=x_{1}$. 
Let $P$ be a set of paths, and define function $\psi: P \rightarrow \Sigma^{\star}$, that extracts from a path $p \in$ $P$, the sequence of events associated with $p$. Thus, if $p=\left(x_{1}, \sigma_{1}, x_{2}, \sigma_{2}, \ldots, \sigma_{l-1}, x_{l}\right)$, then $\psi(p)=\sigma_{1} \sigma_{2} \ldots \sigma_{l-1}$.

The length of a sequence of events $s \in \Sigma^{\star}$ is denoted as $|s|$.

The set of non-negative integers is denoted by $\mathbb{N}$, and the set formed only with 0 and 1 is denoted by $\mathbb{N}_{1}=\{0,1\}$.

The difference between two sets $A$ and $B$ is denoted by $A \backslash B$.

\subsection{Fault detection based on the system fault-free behavior}

In order to deal with the problem of fault detection of large automated systems, whose complete behavior can be very difficult or even impossible to be obtained, mainly the post-fault behavior, some works in the literature propose the identification of the fault-free behavior of the system. The identified model simulates the observed fault-free behavior of the system, i.e., the language generated by the identified model contains all observed sequences of the system, and is used in the fault detection system. The fault detection system compares the sequences of events or the status of the signals of sensors and actuators, and declares the occurrence of a fault when there is a discrepancy between the observed behavior and the predicted behavior described by the identified model.

In this paper, we propose a fault detection scheme based on the identified fault-free behavior of the system. It is important to remark that since the fault detection scheme is based only on the fault-free behavior, it is capable of identifying the occurrence of any fault in the system, and not only predicted faults. The drawback of adopting this strategy is that fault isolation is not carried out by the fault detection scheme. This task can be performed offline, after the fault has been detected, by analyzing the history of sequences of events executed by the system and the status of sensors and actuators. Fault isolation is not addressed in this work.

\section{Discrete-event system identification with the aim of fault detection}

Let us consider the closed-loop system depicted in Figure 2, and assume that the controller has $m_{i}$ binary input signals, $i_{h}$, for $h=1, \ldots, m_{i}$, and $m_{o}$ binary output signals, $o_{h}$, for $h=$ $1, \ldots, m_{o}$. Let vector

$$
u\left(t_{1}\right)=\left[i_{1}\left(t_{1}\right) \ldots i_{m_{i}}\left(t_{1}\right) o_{1}\left(t_{1}\right) \ldots o_{m_{o}}\left(t_{1}\right)\right]^{T},
$$

denote the observation of the controller signals at time instant $t_{1}$. Thus, vector $u\left(t_{1}\right)$ represents the status of the system at a given time instant $t_{1}$. As the system evolves, the status of the system may change due to changes in sensor readings or actuator commands. This leads to the following definition of events of the identified model.

Definition 1 An event of the identified model is any observed instantaneous change in one or more signals of the vector of inputs and outputs of the controller $u$.

Let us consider that a change in at least one of the variables of $u\left(t_{1}\right)$ is observed at time instant $t_{2}$, leading to a different vector of controller signals $u\left(t_{2}\right)$. Then, according to Definition 1 , the set of changes in the binary signals of $u\left(t_{1}\right)$, leading to vector $u\left(t_{2}\right)$, is an event of the identified model. Thus, the set of all events of an identified model, denoted in this 


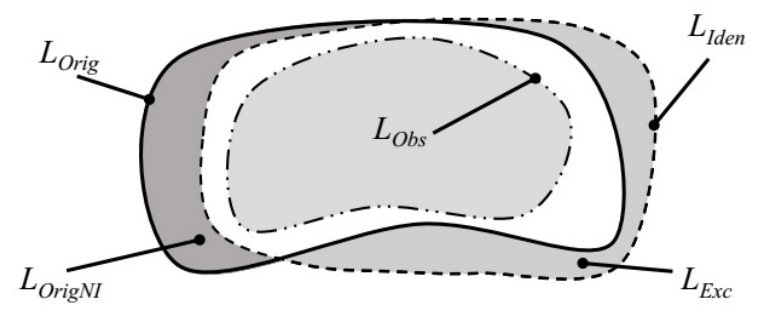

$L_{\text {Orig: }}$ original fault-free language

$L_{\text {Obs }}:$ observed language

$L_{\text {Iden }}:$ identified language

$L_{E x c}$ : exceeding language

$L_{\text {OrigNI }}$ : part of the original language which is non-identified

Fig. 3: Relation between the languages $L_{O r i g}, L_{O b s}, L_{I d e n}, L_{E x c}$, and $L_{O r i g N I}$.

paper as $\Sigma$, is formed of all observed changes in the vector of controller signals $u$. Let $u_{j}$ denote, for simplicity, the status of the system at a given time instant $t_{j}$. Then, the transition from one vector of controller signals $u_{1}$ to another vector $u_{2}$, is represented by the transition $\left(u_{1}, \sigma, u_{2}\right)$, where $\sigma \in \Sigma$. If a sequence of $l$ vectors of controller signals, and the corresponding changes in these signals, is observed, we have an observed path of the system $p=\left(u_{1}, \sigma_{1}, u_{2}, \sigma_{2}, \ldots, \sigma_{l-1}, u_{l}\right)$.

The objective of system identification is to find a model that is capable of describing the observed behavior of the system. Let us consider that the observed paths of the system are denoted as $p_{i}=\left(u_{i, 1}, \sigma_{i, 1}, u_{i, 2}, \sigma_{i, 2}, \ldots, \sigma_{i, l_{i}-1} u_{i, l_{i}}\right)$, for $i=1, \ldots, r$, where $r$ is the number of observed paths, and $l_{i}$ is the number of vertices of each path $p_{i}$. Let us also assume that the system has a unique initial state, associated with $\mathrm{I} / \mathrm{O}$ vector $u_{0}$, and that all paths start at the initial state, i.e., all $\mathrm{I} / \mathrm{O}$ vectors $u_{i, 1}$, for $i=1, \ldots, r$, are equal to $u_{0}$. Thus, associated with each path $p_{i}$ there is a sequence of events $s_{i}=\sigma_{i, 1} \sigma_{i, 2} \ldots \sigma_{i, l_{i}-1}$ and a sequence of output vectors $\omega_{i}=u_{i, 1} u_{i, 2} \ldots u_{i, l_{i}}$. This leads to the following definition of the language observed by the system:

$$
L_{O b s}:=\bigcup_{i=1}^{r} \overline{\left\{s_{i}\right\}} .
$$

It is important to remark that we assume in this paper that none of the paths $p_{i}$ has an associated sequence of events $s_{i}$ that is a prefix of the sequence of events $s_{j}$ of another path $p_{j}$, where $i \neq j$. If this occurs, then path $p_{i}$ does not provide any new information, and can be discarded for system identification.

Since the objective of system identification is to find a model that simulates the observed behavior described by $L_{O b s}$, then the language generated by the identified model, $L_{\text {Iden }}$, must satisfy $L_{O b s} \subseteq L_{I d e n}$. This relation between $L_{O b s}$ and $L_{I d e n}$ is depicted in the diagram of Figure 3.

In a finite time, only part of the sequences of events that the system can generate can be observed, which means that $L_{O b s} \subset L_{\text {Orig }}$, where $L_{\text {Orig }}$ denotes the never known language generated by the system. The relation between the observed language and the original language generated by the system is also described in the diagram of Figure 3.

As it can be seen in Figure 3, two other languages can be defined: (i) $L_{E x c}=L_{I d e n} \backslash L_{\text {Orig }}$; and $(i i) L_{\text {OrigNI }}=L_{\text {Orig }} \backslash L_{\text {Ident }}$. $L_{E x c}$ represents the sequences of events that can be generated by the identified automaton but do not belong to the original behavior of the system. Since the fault detection strategy is based on the observation of events and comparison with the sequences generated by the model, if a sequence of events that is not in the original fault-free system is observed and is in the language of the identified model, then the fault is not detected. Thus, $L_{E x c}$ represents faulty sequences that cannot be detected by the fault detection 
system. On the other hand, $L_{O r i g N I}$ is associated with the sequences that are in the original fault-free system, but are not identified because the paths associated with these sequences have not been observed. The sequences of events of $L_{O \text { rigNI }}$ are associated with false alarms generated by the fault detection system. Clearly, both languages must be reduced in order to obtain an efficient fault detection scheme.

In Klein et al. (2005), it is shown that if a sufficiently large number of vectors of controller signals are observed, then there exists a number $n_{0} \in \mathbb{N}$ such that the difference $L_{\text {Orig }}^{\leq n_{0}} \backslash L_{\text {Obs }}^{\leq n_{0}} \approx \emptyset$, where $L_{\text {Orig }}^{\leq n_{0}}$ and $L_{\text {Obs }}^{\leq n_{0}}$ denote the sets formed with all sequences of events of length smaller than or equal to $n_{0}$ of $L_{O r i g}$ and $L_{O b s}$, respectively. Thus, since $L_{O b s} \subseteq L_{I d e n}$, the subset of $L_{\text {OrigNI }}$ formed with all sequences of events of length smaller than or equal to $n_{0}, L_{\text {OrigNI }}^{\leq n_{0}}$, is also approximately the empty set. Let us assume that $L_{\text {OrigNI }}^{\leq n_{0}}=\emptyset$. Then, all sequences of events of length smaller than or equal to $n_{0}$ that does not belong to the identified model are faulty sequences, and the fault detection system will not raise false alarms. This assumption is formalized as follows.

A1. All paths of length $n_{0}+1$ of the original system are observed, and, consequently, $L_{\text {OrigNI }}^{\leq n_{0}}=\emptyset$.

Assumption A1 being made, the main problem remaining for fault detection and isolation is to reduce the exceeding language. In Klein et al. (2005), the parametric identification algorithm allows to obtain a model satisfying an important property called $k+1$ completeness that guarantees that a sequence of I/O signals $u_{j}$, for $j=1, \ldots, k+1$, where $k$ is the free parameter used for system identification, belongs to the identified NDAAO if, and only if, it belongs to an observed path $p_{i}, i=1, \ldots, r$. By increasing the value of the free parameter $k$, the exceeding language generated by the NDAAO, $L_{E x c}$, reduces, but the size of the model grows. Thus, there is a trade-off to be found between complexity and accuracy of the identified model. Some guidelines to choose appropriately $k$ are given in Klein et al. (2005).

In this paper, an equivalent definition of $k$-completeness, based on sequences of events instead of sequences of observed vectors, is presented. In order to do so, let us first define the set of all observed paths $P:=\left\{p_{i}: i \in R\right\}$, where $R=\{1,2, \ldots, r\}$, and the language formed of all observed subsequences of events of length $n$, as follows:

$$
L_{S, O b s}^{n}:=\left\{s \in \Sigma^{\star}:(|s|=n)\left[\exists i \in R, s \in \operatorname{Sub}\left(\psi\left(p_{i}\right)\right)\right]\right\},
$$

where $\psi: P \rightarrow \Sigma^{\star}$. Then, a model is said to be $k$-complete if for all $n \leq k, L_{S, \text { Obs }}^{n}=L_{S, \text { Ident }}^{n}$, where $L_{S, \text { Ident }}^{n}$ is the set formed of all subsequences of events of the identified model of length $n$.

In the next section, we propose an enhanced model for the identification of DES with the aim of fault detection. The model satisfies the $k$-completeness property, and its exceeding language can be considerably reduced in comparison with the NDAAO for the same value of $k$. Therefore, with the model proposed in this paper, we can obtain accurate and compact models that describe the fault-free system behavior, and that are suitable for fault detection.

\section{Deterministic Automaton with Outputs and Conditional Transitions}

We introduce in this paper a modified automaton model that is suitable for fault detection. The modified automaton is deterministic, with a state output function, and with conditions for the transposition of the transitions associated with the observed paths used to construct the model. This automaton is called Deterministic Automaton with Outputs and Conditional Transitions (DAOCT), and is formally defined as follows. 
Definition 2 A Deterministic Automaton with Outputs and Conditional Transitions (DAOCT) is the nine-tuple:

$$
\mathrm{DAOCT}=\left(X, \Sigma, \Omega, f, \lambda, R, \theta, x_{0}, X_{f}\right),
$$

where $X$ is the set of states, $\Sigma$ is the set of events, $\Omega \subset \mathbb{N}_{1}^{m_{i}+m_{o}}$ is the set of I/O vectors, $f: X \times \Sigma^{\star} \rightarrow X$ is the deterministic transition function, $\lambda: X \rightarrow \Omega$, is the state output function, $R=\{1,2, \ldots, r\}$ is the set of path indices, $\theta: X \times \Sigma \rightarrow 2^{R}$ is the path estimation function, $x_{0}$ is the initial state, and $X_{f} \subseteq X$ is the set of final states.

The sets of events and $\mathrm{I} / \mathrm{O}$ vectors associated with each observed path $p_{i}, i=1, \ldots, r$, are denoted in this paper, respectively, as $\Sigma_{i}$ and $\Omega_{i}$. Thus, the set of events and the set of I/O vectors of the identified model are, respectively, $\Sigma=\cup_{i=1}^{r} \Sigma_{i}$ and $\Omega=\cup_{i=1}^{r} \Omega_{i}$. Each state $x \in X$ of the DAOCT model is associated with an output, given by function $\lambda(x)$, that records the last observed I/O vector. This fact justifies the name Automaton with Outputs of the DAOCT. Since $f$ is a deterministic function, i.e., the occurrence of an event (signal changes in the current $\mathrm{I} / \mathrm{O}$ vector) leads to a unique next state, and, by assumption, the system has only one initial state, then the DAOCT is deterministic. In addition, the transitions must satisfy a condition related with the path estimation function $\theta$ in order to be transposed, which justifies the name Conditional Transitions of the DAOCT.

The DAOCT is obtained from the observed paths $p_{i}, i=1, \ldots, r$, by following the steps of Algorithm 1. As in Klein et al. (2005), a free parameter $k$ is used to construct the identified model, and states and transitions are created in both models in a similar way. Before we present Algorithm 1, let us compute from path $p_{i}$, a path $p_{i}^{k}$ such that the vertices of $p_{i}^{k}$ are sequences of $\mathrm{I} / \mathrm{O}$ vectors of length at most equal to $k$ as follows:

$$
p_{i}^{k}=\left(y_{i, 1}, \sigma_{i, 1}, y_{i, 2}, \sigma_{i, 2}, \ldots, \sigma_{i, l_{i-1}}, y_{i, l_{i}}\right)
$$

where

$$
y_{i, j}=\left\{\begin{array}{l}
\left(u_{i, j-k+1}, \ldots, u_{i, j}\right), \text { if } k \leq j \leq l_{i} \\
\left(u_{i, 1}, \ldots, u_{i, j}\right), \text { if } j<k
\end{array} .\right.
$$

Notice that the sequence of events of $p_{i}^{k}$ is equal to the sequence of events of path $p_{i}$. Thus, the unique difference between $p_{i}$ and $p_{i}^{k}$ is that each vertex of $p_{i}^{k}$ is now associated with a sequence of vectors instead of a single $\mathrm{I} / \mathrm{O}$ vector.

Lemma 1 Each vertex $y_{i, j}$ of path $p_{i}^{k}$ stores the last $(k-1)$ events executed in path $p_{i}^{k}$, if $j \geq k$, and the last $(j-1)$ events, if $j<k$.

Proof. According to Equation 3, each vertex $y_{i, j}$ of path $p_{i}^{k}$ stores the last $k \mathrm{I} / \mathrm{O}$ vectors generated in path $p_{i}^{k}$, if $j \geq k$, and the last $j \mathrm{I} / \mathrm{O}$ vectors if $j<k$. Consequently, the last $(k-1)$ signal changes executed in path $p_{i}^{k}$ are stored in vertex $y_{i, j}$, if $j \geq k$, and the last $(j-1)$ signal changes are stored in $y_{i, j}$, if $j<k$. Since the events $\sigma_{i, j}$ are associated with the signal changes from vector $u_{i, j}$ to $u_{i, j+1}$, then the proof is concluded.

In the following example we illustrate the computation of paths $p_{i}^{k}$ from observed paths $p_{i}, i=1,2, \ldots, r$.

Example 1 Let us consider a system with three binary controller signals, and let us consider the observation of three paths $p_{i}, i=1, \ldots, 3$, given as:

$$
p_{1}=\left(\left[\begin{array}{l}
1 \\
0 \\
0
\end{array}\right], a,\left[\begin{array}{l}
1 \\
1 \\
0
\end{array}\right], b,\left[\begin{array}{l}
0 \\
1 \\
1
\end{array}\right], c,\left[\begin{array}{l}
0 \\
0 \\
0
\end{array}\right], d,\left[\begin{array}{l}
0 \\
0 \\
1
\end{array}\right], e,\left[\begin{array}{l}
1 \\
0 \\
0
\end{array}\right]\right),
$$




$$
\begin{aligned}
& p_{2}=\left(\left[\begin{array}{l}
1 \\
0 \\
0
\end{array}\right], g,\left[\begin{array}{l}
0 \\
0 \\
0
\end{array}\right], h,\left[\begin{array}{l}
1 \\
1 \\
0
\end{array}\right], b,\left[\begin{array}{l}
0 \\
1 \\
1
\end{array}\right], c,\left[\begin{array}{l}
0 \\
0 \\
0
\end{array}\right], i,\left[\begin{array}{l}
1 \\
0 \\
0
\end{array}\right], j,\left[\begin{array}{l}
0 \\
1 \\
1
\end{array}\right], l,\left[\begin{array}{l}
1 \\
0 \\
0
\end{array}\right]\right), \\
& p_{3}=\left(\left[\begin{array}{l}
1 \\
0 \\
0
\end{array}\right], g,\left[\begin{array}{l}
0 \\
0 \\
0
\end{array}\right], h,\left[\begin{array}{l}
1 \\
1 \\
0
\end{array}\right], b,\left[\begin{array}{l}
0 \\
1 \\
1
\end{array}\right], i,\left[\begin{array}{l}
1 \\
1 \\
1
\end{array}\right], m,\left[\begin{array}{l}
0 \\
0 \\
0
\end{array}\right], d,\left[\begin{array}{l}
0 \\
0 \\
1
\end{array}\right], n,\left[\begin{array}{l}
1 \\
1 \\
0
\end{array}\right]\right),
\end{aligned}
$$

where each event is associated with the rising or the falling edge of the controller signals. For instance, $a$ denotes the rising edge of the second controller signal, and $b$ denotes the falling edge of the first controller signal and the rising edge of the third controller signal.

According to Equations (2) and (3), and choosing the free parameter $k=2$, we obtain the following modified paths $p_{1}^{2}, p_{2}^{2}$ and $p_{3}^{2}$ :

$$
\begin{gathered}
p_{1}^{2}=\left(\left[\begin{array}{l}
1 \\
0 \\
0
\end{array}\right], a,\left[\begin{array}{ll}
1 & 1 \\
0 & 1 \\
0 & 0
\end{array}\right], b,\left[\begin{array}{ll}
1 & 0 \\
1 & 1 \\
0 & 1
\end{array}\right], c,\left[\begin{array}{ll}
0 & 0 \\
1 & 0 \\
1 & 0
\end{array}\right], d,\left[\begin{array}{ll}
0 & 0 \\
0 & 0 \\
0 & 1
\end{array}\right], e,\left[\begin{array}{ll}
0 & 1 \\
0 & 0 \\
1 & 0
\end{array}\right]\right), \\
p_{2}^{2}=\left(\left[\begin{array}{l}
1 \\
0 \\
0
\end{array}\right], g,\left[\begin{array}{ll}
1 & 0 \\
0 & 0 \\
0 & 0
\end{array}\right], h,\left[\begin{array}{ll}
0 & 1 \\
0 & 1 \\
0 & 0
\end{array}\right], b,\left[\begin{array}{ll}
1 & 0 \\
1 & 1 \\
0 & 1
\end{array}\right], c,\left[\begin{array}{ll}
0 & 0 \\
1 & 0 \\
1 & 0
\end{array}\right], i,\left[\begin{array}{ll}
0 & 1 \\
0 & 0 \\
0 & 0
\end{array}\right], j,\left[\begin{array}{ll}
1 & 0 \\
0 & 1 \\
0 & 1
\end{array}\right], l,\left[\begin{array}{ll}
0 & 1 \\
1 & 0 \\
1 & 0
\end{array}\right]\right), \\
p_{3}^{2}=\left(\left[\begin{array}{l}
1 \\
0 \\
0
\end{array}\right], g,\left[\begin{array}{ll}
1 & 0 \\
0 & 0 \\
0 & 0
\end{array}\right], h,\left[\begin{array}{ll}
0 & 1 \\
0 & 1 \\
0 & 0
\end{array}\right], b,\left[\begin{array}{ll}
1 & 0 \\
1 & 1 \\
0 & 1
\end{array}\right], i,\left[\begin{array}{ll}
0 & 1 \\
1 & 1 \\
1 & 1
\end{array}\right], m,\left[\begin{array}{ll}
1 & 0 \\
1 & 0 \\
1 & 0
\end{array}\right], d,\left[\begin{array}{ll}
0 & 0 \\
0 & 0 \\
0 & 1
\end{array}\right], n,\left[\begin{array}{ll}
0 & 1 \\
0 & 1 \\
1 & 0
\end{array}\right]\right) .
\end{gathered}
$$

In order to present the algorithm for the computation of the identified DAOCT, it is also necessary to define the labeling function $\tilde{\lambda}: X \rightarrow \Omega^{k}$, where $\Omega^{k}$ is formed of all sequences of symbols of $\Omega$ of length smaller than or equal to $k$. Function $\tilde{\lambda}$ associates to each state $x \in X$, a sequence of symbols $\omega^{k} \in \Omega^{k}$. Let $\tilde{\lambda}_{l}(x)$ denote the last output vector of $\tilde{\lambda}(x)$.

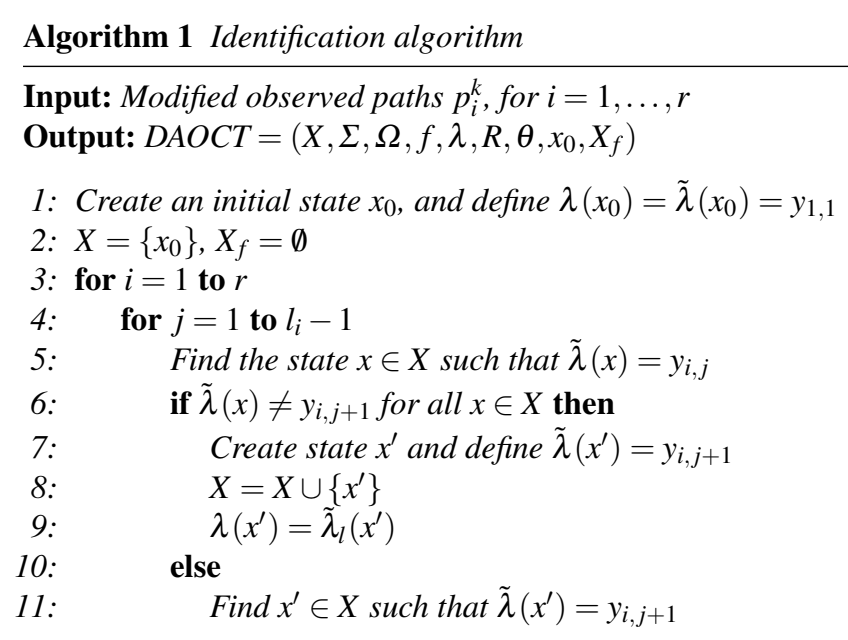




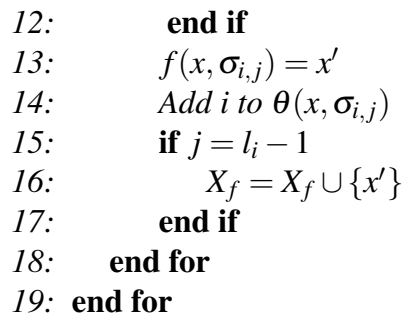

Each transition $x^{\prime}=f(x, \sigma)$ of automaton DAOCT has a corresponding set $\theta(x, \sigma)$ of indices that is associated with the paths $p_{i}$ that contain transition $\left(x, \sigma, x^{\prime}\right)$. Function $\theta$ is used in the DAOCT evolution rule to provide a path estimator, such that if the paths associated with a transition are not coherent with the paths of the observed sequence of events, then the transition is not enabled. This fact is clearly presented in the definition of the language generated by the DAOCT. In order to present the language generated by the DAOCT, it is first necessary to extend the domain of function $\theta$ to consider the execution of sequences of events, obtaining the extended path estimation function $\theta_{s}: X \times \Sigma^{\star} \rightarrow 2^{R}$. $\theta_{s}$ can be defined recursively as:

$$
\begin{aligned}
& \theta_{s}(x, \varepsilon)=R, \\
& \theta_{s}(x, s \sigma)=\left\{\begin{array}{l}
\theta_{s}(x, s) \cap \theta\left(x^{\prime}, \sigma\right), \text { where } x^{\prime}=f(x, s), \text { if } f(x, s \sigma) ! \\
\text { undefined, otherwise. }
\end{array}\right.
\end{aligned}
$$

The language generated by the DAOCT is given by

$$
L(\mathrm{DAOCT}):=\left\{s \in \Sigma^{\star}: f\left(x_{0}, s\right) ! \wedge \theta_{s}\left(x_{0}, s\right) \neq \emptyset\right\} .
$$

Notice that a sequence of events $s$ is only feasible in the DAOCT, if $f\left(x_{0}, s\right)$ is defined, and there is at least one path in the path estimate after the occurrence of $s$, represented by condition $\theta_{s}\left(x_{0}, s\right) \neq \emptyset$.

It is also possible to define the language formed of all subsequences of events of length $n$ generated by the DAOCT as follows:

$$
L_{S}^{n}(\mathrm{DAOCT}):=\left\{s \in \Sigma^{\star}:(|s|=n)\left[\exists x_{i} \in X, f\left(x_{i}, s\right) ! \wedge \theta_{s}\left(x_{i}, s\right) \neq \emptyset\right]\right\} .
$$

Example 2 Let us now compute the DAOCT obtained according to Algorithm 1 considering the observed paths $p_{i}, i=1,2,3$, presented in Example 1. In Figures 4 and 5, we present the DAOCT obtained by choosing $k=1$ and $k=2$, respectively. The final states are represented by double circles. Notice that each transition is labeled with an event from $\Sigma$, and a set associated with the paths $p_{i}^{k}$ where each transition is defined, i.e., each transition $\left(x, \sigma, x^{\prime}\right)$, where $x^{\prime}=f(x, \sigma)$, of the DAOCT is labeled with $\sigma$ and $\theta(x, \sigma)$. In addition, notice that, for $k=2$, the corresponding DAOCT is acyclic.

Remark 1 Notice, according to Algorithm 1, that, in the worst-case, the number of states of the DAOCT model is equal to the sum of the number of vertices of all paths $p_{i}, i=1, \ldots, r$, used in the identification procedure. Thus, in the worst-case, $|X|=\sum_{i=1}^{r} l_{i}$. This result can be achieved by choosing $k \geq \max _{i \in\{1, \ldots, r\}} l_{i}$. In practice, a small value of $k$ can be used to accurately describe the observed behavior of the system, which leads to smaller identified models for fault detection. In addition, it can be seen that even for $k=1$, in the worstcase, the number of states of the model grows exponentially with the number of inputs, 


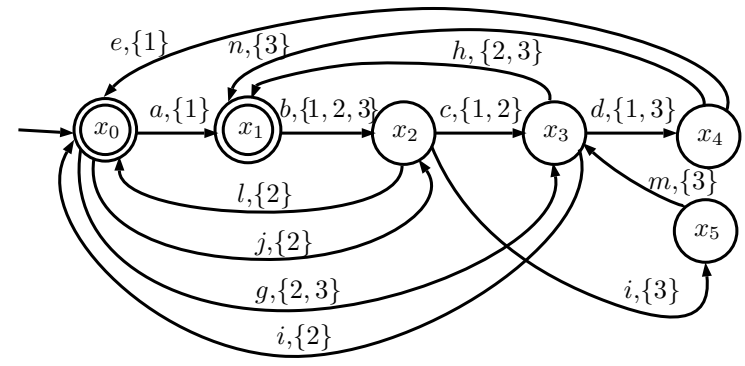

Fig. 4: DAOCT computed considering $k=1$.

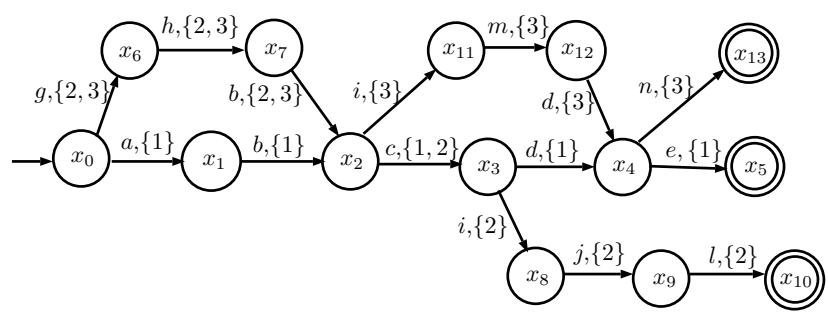

Fig. 5: DAOCT computed considering $k=2$.

$m_{i}$, and outputs, $m_{o}$, of the controller, i.e., $|X|=2^{\left(m_{i}+m_{o}\right)}$. However, the exponential growth of the model size only occurs if all possible I/O binary vectors are observed in the faultfree behavior of the system. Nevertheless, this is, in general, a very conservative bound for the model size. In practice, due to the closed-loop between plant and controller, physical constraints of the plant forbid to reach lots of the possible combinations of the signal values of the controller I/O vectors. Moreover, the identified paths correspond to the controlled behavior of the system, that is reduced in comparison with the behavior of the uncontrolled plant, since the controller is designed to satisfy the system specifications. This also naturally reduces the number of observed $\mathrm{I} / \mathrm{O}$ vectors for identification.

The following theorem shows that the observed fault-free language $L_{O b s}$ is a subset of the language generated by the DAOCT, $L(\mathrm{DAOCT})$, i.e., the identified DAOCT simulates the observed fault-free language of the system.

Theorem $1 L_{O b s} \subseteq L(D A O C T)$.

Proof. Let $s=\sigma_{i, 1} \sigma_{i, 2} \ldots \sigma_{i, l_{i}-1}$ be a sequence of events of an observed path $p_{i}^{k}=\left(y_{i, 1}, \sigma_{i, 1}\right.$, $\left.y_{i, 2}, \sigma_{i, 2}, \ldots, \sigma_{i, l_{i-1}}, y_{i, l_{i}}\right)$. According to Algorithm 1 , there is a path in the DAOCT $\left(x_{1}, \sigma_{i, 1}, x_{2}\right.$, $\left.\sigma_{i, 2}, \ldots, \sigma_{i, l_{i-1}}, x_{l_{i}}\right)$, associated with $p_{i}^{k}$, where $x_{i}$ is not necessarily distinct from $x_{j}$, for $i \neq j$, $i, j=1,2, \ldots, l_{i}$, and $i \in \theta\left(x_{j}, \sigma_{i, j}\right)$, for $j=1, \ldots, l_{i}-1$. Thus, any prefix of $s$ belongs to the language generated by DAOCT, which implies that $L_{O b s} \subseteq L(\mathrm{DAOCT})$.

In the sequel, we prove that the identified model is $k$-complete.

Theorem 2 For a given value of $k$, the identified DAOCT is $k$-complete, i.e., $L_{S}^{n}(D A O C T)=$ $L_{S, \text { Obs }}^{n}$, for all $n \leq k$. 
Proof. Since, according to Theorem 1, L(DAOCT) $\supseteq L_{O b s}$, then $L_{S}^{n}(\mathrm{DAOCT}) \supseteq L_{S, O b s}^{n}$, for all $n \leq k$. Let us now prove that $L_{S}^{n}(\mathrm{DAOCT}) \subseteq L_{S, O b s}^{n}$. Let $p=\left(x_{q}, \sigma_{q}, x_{q+1}, \sigma_{q+1}\right.$, $\left.\ldots, \sigma_{q+n}, x_{q+n+1}\right)$ be a feasible path of the DAOCT of length $n+1$, i.e., $\theta_{s}\left(x_{q}, \sigma_{q} \ldots \sigma_{q+n}\right) \neq$ $\emptyset$. According to Algorithm 1, any transition of $p$ is associated with a transition in at least one path $p_{i}^{k}, i=1, \ldots, r$. Let us consider the last transition of $p,\left(x_{q+n}, \sigma_{q+n}, x_{q+n+1}\right)$, and assume that $\left(\tilde{\lambda}\left(x_{q+n}\right), \sigma_{q+n}, \tilde{\lambda}\left(x_{q+n+1}\right)\right)$ is the transition in path $p_{x}^{k}$, where $x \in\{1,2, \ldots, r\}$, associated with transition $\left(x_{q+n}, \sigma_{q+n}, x_{q+n+1}\right)$. Let $\tilde{\lambda}\left(x_{q+n}\right)=y_{x, q+n}$. According to Lemma 1, all suffixes of length $1, \ldots, k-1$ of the sequences that reach $y_{x, q+n}$ must also belong to $p_{x}^{k}$. Consequently, $\sigma_{q} \sigma_{q+1} \ldots \sigma_{q+n} \in L_{S, O b s}^{n}$, for all $n \leq k$, which implies that $L_{S}^{n}(\mathrm{DAOCT}) \subseteq L_{S, O b s}^{n}$, for $n \leq k$.

It is important to remark that $L_{E x c}=L(\mathrm{DAOCT}) \backslash L_{\text {Orig }}$ can be different from the empty set, which means that the fault detection system may be not capable of identifying all system faults. However, this exceeding language is smaller than the exceeding language $L($ NDAAO $) \backslash L_{\text {Orig }}$, i.e., the exceeding language that is obtained using the model proposed in Klein et al. (2005). Thus, the enhanced model proposed in this paper reduces the number of non-detectable faults in comparison with the NDAAO. In the next theorem we show that if the DAOCT does not have cyclic paths, then $L_{E x c}=\emptyset$.

Theorem 3 If the identified DAOCT does not have cyclic paths for a given value of $k$, then $L_{E x c}=\emptyset$.

Proof. Notice, according to Algorithm 1, that each transition of the DAOCT is associated with at least one observed path $p_{i}, i=1, \ldots, r$. Moreover, since all events of path $p_{i}, i=$ $1, \ldots, r$ are observable, then, associated with each path $p_{i}$, there is a number $n_{i}<l_{i}$ such that $p_{i}$ can be distinguished from all other paths after the observation of $n_{i}$ events. Consequently, since the DAOCT does not have cyclic paths, then, after the occurrence of the observed sequence of events $s_{i}=\psi\left(p_{i}\right)$, we have that $\theta\left(x_{0}, s_{i}\right)=\{i\}$. In addition, since the DAOCT is acyclic, the intersection of the path estimates of two transitions leaving the same state of the DAOCT must be empty, which implies that all paths $p_{i}$ are uniquely determined before reaching its corresponding final state. Thus, if a sequence $s \in \Sigma^{\star} \backslash L_{O b s}$ is observed, then two possibilities may happen: $(i) f\left(x_{0}, s\right)$ is not defined; or $(i i) f\left(x_{0}, s\right)$ is defined, but $\theta_{s}\left(x_{0}, s\right)=\emptyset$. Thus, $s \notin L(\mathrm{DAOCT})$, which implies that $L(\mathrm{DAOCT}) \subseteq L_{O b s}$, and, therefore, $L_{E x c}=\emptyset$.

Let us introduce the language generated by the DAOCT formed with all traces of length smaller than or equal to a given value $n$ as follows:

$$
L^{\leq n}(\mathrm{DAOCT}):=\left(\bigcup_{i=0}^{n} L_{S}^{i}(\mathrm{DAOCT})\right) \cap L(\mathrm{DAOCT}) .
$$

According to Theorem 3, if $k$ is chosen such that the DAOCT does not have cyclic paths, then, $L_{E x c}=L(\mathrm{DAOCT}) \backslash L_{\text {Orig }}=\emptyset$, and there is no non-detectable faults. In addition, if Assumption A1 also holds, the observed language $L_{\mathrm{Obs}}^{\leq n_{0}}$ is equal to the original system language $L_{\text {Orig }}^{\leq n_{0}}$, and there is no false alarms for all observed traces of length smaller than or equal to $n_{0}$. Thus, under both assumptions, $L^{\leq n_{0}}$ (DAOCT) $=L_{\text {Orig }}^{\leq n_{0}}$. It is important to remark that, since the NDAAO model does not use the path estimation function, then, even if it is acyclic, sequences that have not been observed can be generated by the identified model. Thus, Theorem 3 does not hold true if a NDAAO model is used.

Let us now define the subset of the exceeding language $L_{E x c}$ formed with all sequences of length smaller than or equal to $n$ as $L_{\bar{E} x c}^{\leq n}=L^{\leq n}$ (DAOCT) $\backslash L_{\text {Orig }}^{\leq n}$. If we consider that $n \leq$ $n_{0}$, then, according to Assumption A1, $L_{E x c}^{\leq n}$ can be rewritten as $L_{E x c}^{\leq n}=L^{\leq n}(\mathrm{DAOCT}) \backslash L_{O b s}^{\leq n}$. 
In the following example we compare the exceeding language $L_{\bar{E} x c}^{\leq n}$, for different values of $n \leq n_{0}$, generated by the DAOCT model proposed in this paper with the NDAAO model presented in Klein et al. (2005).

Example 3 In Figure 6, we compare the cardinality of the exceeding language $L_{\bar{E} x c}^{\leq n}$, generated by the DAOCT model (o) of Example 2, with the exceeding language generated by the NDAAO model $(*)$, for $k=1$. Notice that the exceeding language is greatly reduced using the DAOCT model in comparison with the NDAAO model. Moreover, in this case, both models have the same number of states (6 states). This shows that the DAOCT model is more appropriate for fault detection then the NDAAO model proposed in Klein et al. (2005).

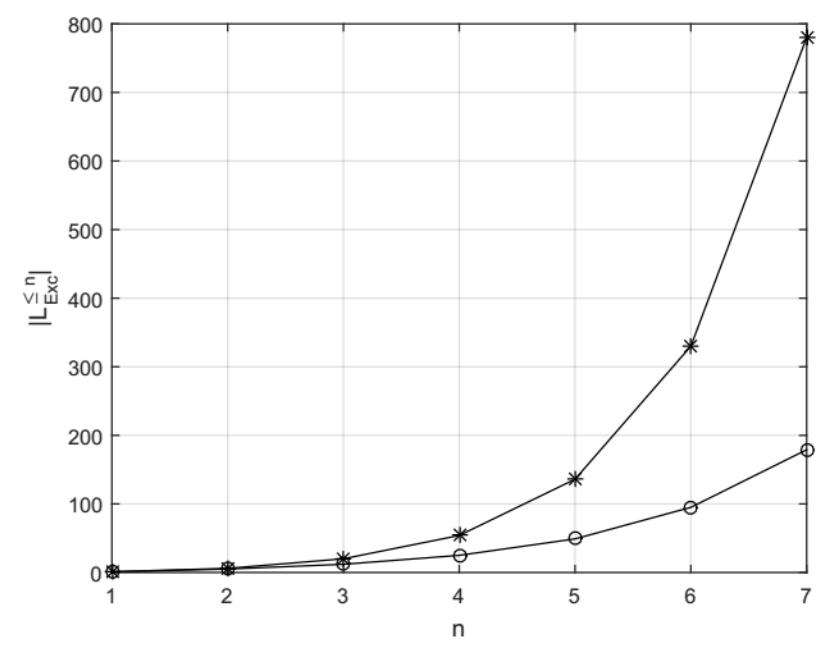

Fig. 6: Comparison between the cardinality of the exceeding language generated by the DAOCT (o) and $\operatorname{NDAAO}(*)$ models, computed considering $k=1$, for different values of $n$.

It is important to remark that, since, as shown in Example 2, the DAOCT model does not have cyclic paths for $k=2$, then, in accordance with Theorem 3, the exceeding language generated by the DAOCT model for $k=2$ is empty for all values of $n$.

\section{Example}

In this section, a practical example is used to illustrate the identification method proposed in the paper. The plant is simulated using the software FACTORY I/O (Real Games, 2018), and is controlled by a PLC of the manufacturer Schneider Electric, as shown in Figure 7. The communication between the PLC and the computer where the plant is simulated is carried out using the Advantech data acquisition board USB-4750. FACTORY I/O is a 3D simulation software for learning automation technologies, and it allows to construct a virtual factory using a selection of common industrial parts. After the construction of the digital system, it can be controlled by a PLC, and the input and output signals of the controller can be used to identify the fault-free behavior of the system. 


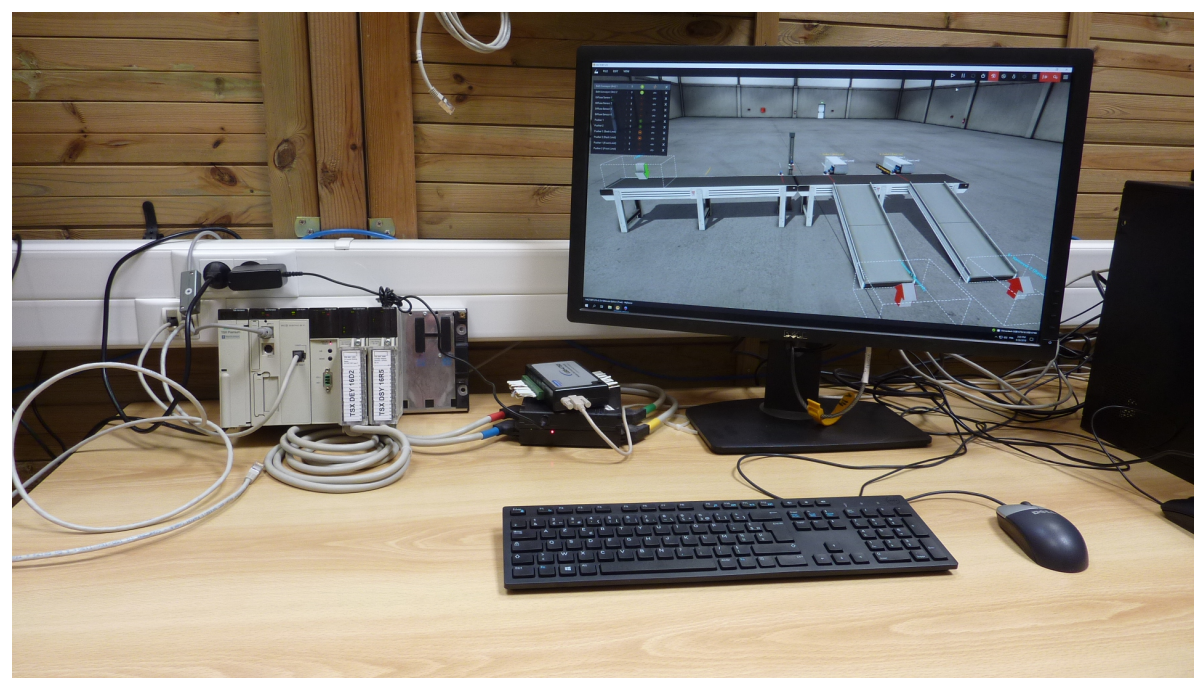

Fig. 7: Simulation setup.

The virtual plant is depicted in Figure 8 and consists of a sorting unit system. The objective of this system is to sort parcels, that are randomly delivered to the feeder conveyor, according to their size. Two sensors, located at the end of the feeder conveyor, $k_{1}$ and $k_{2}$, inform the presence of a parcel and its corresponding size. If the parcel is a small one then the falling edge of sensor $k_{1}$ is observed without observing $k_{2}=1$, and if the parcel is a big one, we observe $k_{1}=1$ and $k_{2}=1$. The first pusher in the distribution conveyor, Pusher 1 , send small parcels to the first slide, and big parcels are sent to the second slide by Pusher 2 . When the distribution conveyor has a parcel, and another parcel arrives at the end of the feeder conveyor $\left(k_{1}=1\right)$, the feeder conveyor is stopped and is turned on again only when the parcel of the distribution conveyor is sorted. When a small (resp. big) parcel is in front of Pusher 1 (resp. Pusher 2), detected by the falling edge of the signal of the sensor located at the side of Pusher 1 (resp. Pusher 2), sensor $k_{3}$ (resp. $k_{4}$ ), the distribution conveyor is turned off, and is turned on again only after the end of the movement of Pusher 1 (resp. Pusher 2). Each pusher has two sensors to indicate if it is completely retracted or extended, sensors $k_{5}$ and $k_{6}$ for Pusher 1 , and sensors $k_{7}$ and $k_{8}$ for Pusher 2. Thus, the controller of this system has 8 inputs (corresponding to the 8 sensors) and 4 outputs (corresponding to the 4 actuators), which implies that each I/O vector $u_{j}$ has 12 binary entries.

In order to obtain the fault-free behavior of the system depicted in Figure 8, simulations have been carried out using the software FACTORY I/O, guaranteeing that the system is completely free of faults. Based on the simulation of the fault-free behavior of the plant, thirteen cyclic fault-free paths $p_{i}, i=1, \ldots, 13$, have been identified, and we have computed the DAOCT and the NDAAO models for $k=1$ and $k=2$. In Figure 9, we compare the cardinality of the exceeding language $L_{\overline{E x c}, N D A A O}^{\leq n}$ of the NDAAO model with the cardinality of the exceeding language $L_{E x c, D A O C T}^{\leq n}$ of the DAOCT model for $k=1$, for different values of $n$. As it can be seen from Figure 9, the exceeding language of the DAOCT model is greatly reduced in comparison with the exceeding language of the NDAAO model for large values of $n$. In Figure 10, we compare the exceeding language of the NDAAO model with the exceeding language of the DAOCT model for $k=2$. In this case, $L_{E x c, D A O C T}^{\leq n}$ is empty for 


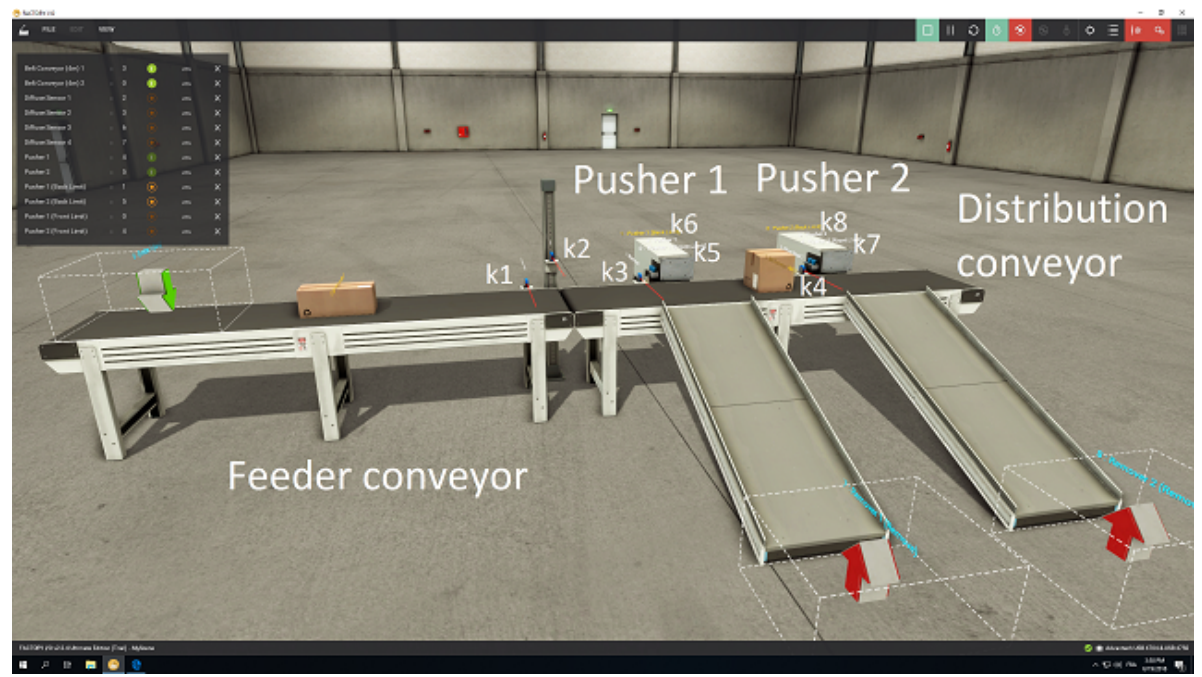

Fig. 8: Sorting unit system simulated using software FACTORY I/O.

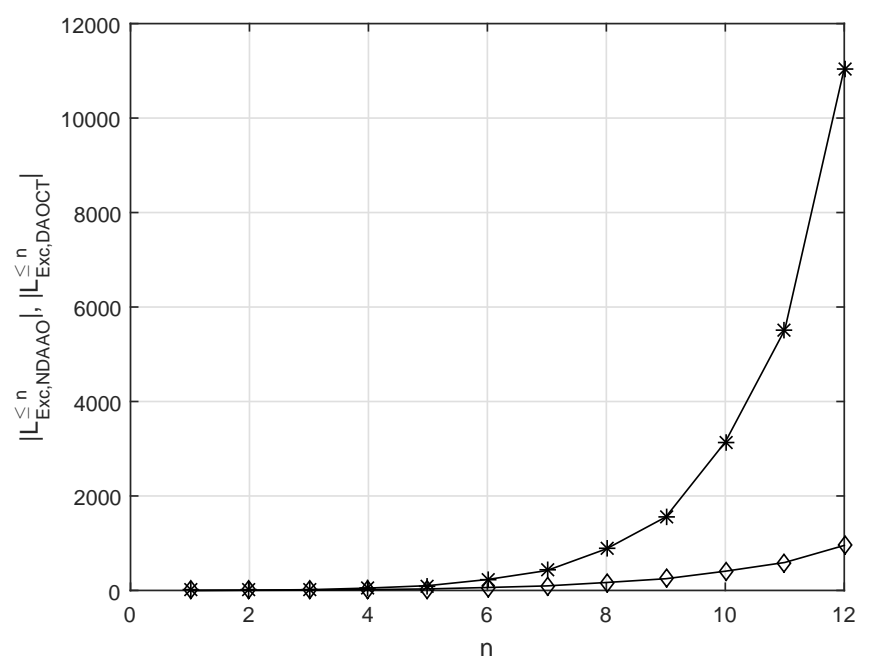

Fig. 9: Exceeding language generated by the DAOCT $(\diamond)$ and NDAAO $(*)$ models, computed considering $k=1$ for the simulated sorting unit system.

all values of $n$, which shows that the DAOCT model represents all, and only all, fault-free paths that have been observed for the identification of the model, reducing the number of non-detectable fault occurrences.

The number of states of the DAOCT is 34 and 51 for $k=1$ and $k=2$, respectively, while the number of states of the NDAAO is 34 and 40 for $k=1$ and $k=2$, respectively. Although the NDAAO leads to more compact models than the DAOCT for the same value of $k$, the 


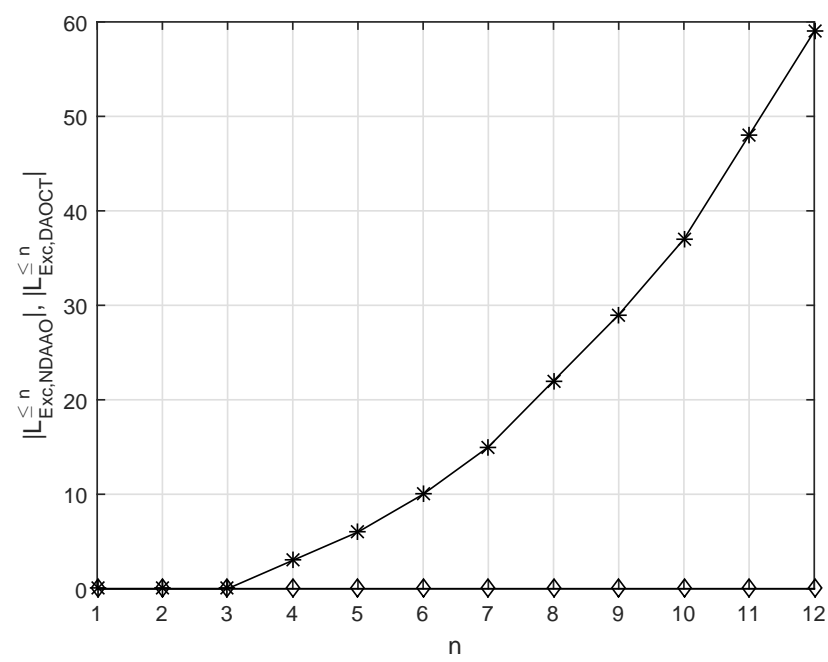

Fig. 10: Exceeding language generated by the DAOCT $(\diamond)$ and NDAAO $(*)$ models, computed considering $k=2$ for the simulated sorting unit system.

exceeding language is much larger using the NDAAO model, which shows that the DAOCT model is more suitable for fault detection than the NDAAO model.

In order to obtain the DAOCT model, we have implemented Algorithm 1 using Matlab in a computer Intel Core i7, with $2.4 \mathrm{GHz}$ and $8 \mathrm{~GB}$ RAM, and the time required for the computation of the DAOCT model is only 0.19 seconds, for $k=1$, and 0.32 seconds, for $k=2$. This shows that the computation of the DAOCT model is not time consuming, and can be efficiently carried out by using Algorithm 1.

\section{Conclusions}

We present, in this paper, a new model for the identification of the fault-free behavior of discrete event systems for fault detection, called DAOCT model. The DAOCT model uses more information for fault detection than other models proposed in the literature and, therefore, leads to a smaller exceeding language. In order to illustrate the advantages of the proposed method, the model of a virtual plant, simulated using a 3D simulation software and controlled by a programmable logic controller, is identified, and the exceeding language generated by the identified model is compared with the exceeding language of other model presented in the literature, called NDAAO model. It is shown that the exceeding language of the DAOCT model is greatly reduced in comparison with the NDAAO model, without a significant increase in the number of states.

\section{References}

Boschert, S. and Rosen, R. (2016). Digital Twin - The Simulation Aspect. In Hehenberger P., Bradley D. (eds) Mechatronic Futures, pages 59-74. Springer, Cham. 
Cabasino, M. P., Darondeau, P., Fanti, M. P., and Seatzu, C. (2013). Model identification and synthesis of discrete-event systems. In Contemporary Issues in System Science and Engineering. Wiley.

Cabasino, M. P., Giua, A., Hadjicostis, C. N., and Seatzu, C. (2014). Fault model identification and synthesis in petri nets. Discrete Event Dynamic Syst., 24(3):275-307.

Cabasino, M. P., Giua, A., and Seatzu, C. (2007). Identification of petri nets from knowledge of their language. Discrete Event Dynamic Syst., 17(4):447-474.

Cabral, F. G. and Moreira, M. V. (2017). Synchronous codiagnosability of modular discreteevent systems. In IFAC World Congress, pages 7025-7030, Toulouse, France. IFAC.

Cabral, F. G., Moreira, M. V., and Diene, O. (2015a). Online fault diagnosis of modular discrete-event systems. In Decision and Control (CDC), 2015 IEEE 54th Annual Conference on, pages 4450-4455. IEEE.

Cabral, F. G., Moreira, M. V., Diene, O., and Basilio, J. C. (2015b). A Petri net diagnoser for discrete event systems modeled by finite state automata. IEEE Transactions on Automatic Control, pages 59-71.

Carvalho, L. K., Basilio, J. C., and Moreira, M. V. (2012). Robust diagnosis of discrete-event systems against intermittent loss of observations. Automatica, 48(9):2068-2078.

Carvalho, L. K., Moreira, M. V., Basilio, J. C., and Lafortune, S. (2013). Robust diagnosis of discrete-event systems against permanent loss of observations. Automatica, 49(1):223231.

Cassandras, C. and Lafortune, S. (2008). Introduction to Discrete Event System. Springer, New York, 2nd edition.

Debouk, R., Lafortune, S., and Teneketzis, D. (2000). Coordinated decentralized protocols for failure diagnosis of discrete event systems. Discrete Event Dynamic Systems: Theory and Applications, 10(1):33-86.

Dotoli, M., Fanti, M. P., and Mangini, A. M. (2008). Real time identification of discrete event systems using petri nets. Automatica, 44(5):1209-1219.

Dotoli, M., Fanti, M. P., Mangini, A. M., and Ukovich, W. (2011). Identification of the unobservable behaviour of industrial automation systems by petri nets. Control Engineering Practice, 19:958-966.

Estrada-Vargas, A. P., Lesage, J.-J., and López-Mellado, E. (2014a). A stepwise method for identification of controlled discrete manufacturing systems. Int. J. Comput. Integr. Manuf., 28(2):187-199.

Estrada-Vargas, A. P., López-Mellado, E., and Lesage, J.-J. (2010). A comparative analysis of recent identification approaches for discrete-event systems. Math. Probl. Eng., 2010:121.

Estrada-Vargas, A. P., López-Mellado, E., and Lesage, J.-J. (2014b). Input-output identification of controlled discrete manufacturing systems. Int. J. Syst. Sci., 45(3):456-471.

Estrada-Vargas, A. P., López-Mellado, E., and Lesage, J.-J. (2015). A black-box identification method for automated discrete event systems. IEEE Transactions on Automation Science and Engineering, 14(3):1321-1336.

Glaessgen, E. H. and Stargel, D. (2012). The digital twin paradigm for future NASA and U.S. Air Force vehicles. In 53rd Structures, Structural Dynamics, and Materials Conference, pages 1-14. IEEE.

Grieves, M. and Vickers, J. (2017). Digital Twin: Mitigating Unpredictable, Undesirable Emergent Behavior in Complex Systems. In: Kahlen FJ., Flumerfelt S., Alves A. (eds) Transdisciplinary Perspectives on Complex Systems, pages 85-113. Springer, Cham.

Klein, S., Litz, L., and Lesage, J.-J. (2005). Fault detection of discrete event systems using an identification approach. In 16th IFAC World Congress, pages 92-97, Prague, Czech 
Republic.

Lefebvre, D. and Delherm, C. (2007). Diagnosis of des with petri net models. IEEE Transactions on Automation Science and Engineering, 4(1):114âĂŞ118.

Medhi, S. O. E., Leclercq, E., and Lefebvre, D. (2006). Petri nets design and identification for the diagnosis of discrete event systems. In IAR Annu. Meeting. IEEE.

Moor, T., Raisch, J., and Young, S. (1998). Supervisory control of hybrid systems via 1complete approximations. In Proceedings of the IEEE Workshop on Discrete-Event Systems, pages 426-431, Cagliari, Italy.

Moreira, M. V., Jesus, T. C., and Basilio, J. C. (2011). Polynomial time verification of decentralized diagnosability of discrete event systems. IEEE Transactions on Automatic Control, pages 1679-1684.

Moreira, M. V. and Lesage, J.-J. (2018). Enhanced discrete event model for system identification with the aim of fault detection. In 14th Workshop on Discrete Event Systems, pages 172-178, Sorrento Coast, Italy. IFAC.

Qiu, W. and Kumar, R. (2006). Decentralized failure diagnosis of discrete event systems. IEEE Transactions on Systems, Man, and Cybernetics Part A:Systems and Humans, 36(2):384-395.

Real Games (2018). FACTORY I/O. http://factoryio.com, accessed in 06-26-2018.

Roth, M., Lesage, J.-J., and Litz, L. (2009). An FDI method for manufacturing systems based on an identified model. In 13th IFAC Symposium on Information Control Problems in Manufacturing (INCOM2009), pages 1406-1411, Moscow, Russia.

Roth, M., Lesage, J.-J., and Litz, L. (2011). The concept of residuals for fault localization in discrete event systems. Control Engineering Practice, 19(9):978-988.

Sampath, M., Sengupta, R., Lafortune, S., Sinnamohideen, K., and Teneketzis, D. (1995). Diagnosability of discrete-event systems. IEEE Trans. on Automatic Control, 40(9):15551575.

Santoro, L. P. M., Moreira, M. V., and Basilio, J. C. (2017). Computation of minimal diagnosis bases of discrete-event systems using verifiers. Automatica, 77:93-102.

Schleicha, B., Anwer, N., Mathieu, L., and Wartzacka, S. (2017). Shaping the digital twin for design and production engineering. CIRP Annals, 66(1):141-144.

Uhlemann, T. H.-J., Lehmann, C., and Steinhilper, R. (2017). The digital twin: Realizing the cyber-physical production system for industry 4.0. Procedia CIRP, 61:335-340.

Zaytoon, J. and Lafortune, S. (2013). Overview of fault diagnosis methods for discrete event systems. Annual Reviews in Control, pages 308-320. 